\title{
Solid lipid nanoparticles with TPGS and Brij 78: A co-delivery vehicle of curcumin and piperine for reversing P-glycoprotein-mediated multidrug resistance in vitro
}

\author{
JINGLING TANG $^{1^{*}}$, HONGYU JI ${ }^{2 *}$, JINMEI REN ${ }^{1,2}$, MENGTING LI ${ }^{1,2}$, NANNAN ZHENG ${ }^{1}$ and LINHUA WU ${ }^{1,2}$ \\ ${ }^{1}$ Department of Pharmaceutics, School of Pharmacy, Harbin Medical University; \\ ${ }^{2}$ Department of Pharmacy, The Second Affiliated Hospital, Harbin Medical University, \\ Key Laboratory of Medications Research, College of Heilongjiang Province, Harbin, Heilongjiang 150086, P.R. China
}

Received June 23, 2015; Accepted October 24, 2016

DOI: $10.3892 / \mathrm{ol} .2016 .5421$

\begin{abstract}
Multidrug resistance (MDR) is a main clinical hurdle for chemotherapy of cancer, and overexpression of $\mathrm{P}$-glycoprotein (P-gp) is a key factor. In the present study, a new co-delivery system for reversing MDR was designed and developed. The system was composed of curcumin (Cur) and piperine (Pip) encapsulated in solid lipid nanoparticles (SLNs) with tocopheryl polyethylene glycol succinate (TPGS) and Brij 78 [(Cur+Pip)-SLNs]. TPGS and Brij 78 could sensitize MDR tumors by inhibiting the P-gp drug efflux system. The combination of Cur and Pip, when administered in SLNs formulations, resulted in a significant enhancement in cytotoxicity and allowed efficient intracellular delivery of the drugs in drug-resistant A2780/Taxol cells. This dual inhibitory strategy may have significant potential in the clinical management of MDR in cancer.
\end{abstract}

\section{Introduction}

Multidrug resistance (MDR) is a major hurdle for successful chemotherapy, and accounts for failure in $>90 \%$ of patients $(1,2)$. The mechanisms of MDR have been well documented in the literature $(1,2)$. Of all these mechanisms, overexpression of P-glycoprotein (P-gp), which is encoded by the MDR1 gene, is the most typical and commonly encountered theory. $\mathrm{P}$-gp is an important transporter protein belonging to the

Correspondence to: Professor Linhua Wu, Department of Pharmacy, The Second Affiliated Hospital, Harbin Medical University, Key Laboratory of Medications Research, College of Heilongjiang Province, 157 Baojian Road, Harbin, Heilongjiang 150086, P.R. China

E-mail: yaoji636@hotmail.com

"Contributed equally

Key words: curcumin, multidrug resistance, piperine, P-glycoprotein, solid lipid nanoparticles adenosine triphosphate (ATP)-binding cassette (ABC) family of membrane transporters. Due to its participation in the efflux of numerous cytotoxic anticancer drugs from tumor cells, $\mathrm{P}-\mathrm{gp}$ could directly influence the pharmacokinetics and pharmacodynamics of these drugs (3). As a result, it has become a severe challenge to overcome P-gp-mediated drug resistance.

Curcumin (Cur), a hydrophobic polyphenol derived from the rhizome of the herb Curcuma longa, has a wide spectrum of biological and pharmacological activities. Cur has been shown to exhibit antioxidant, anti-inflammatory, antimicrobial and anticarcinogenic activities (4). The anticancer properties of Cur have been primarily attributed by its ability to block the transcriptional factor nuclear factor $(\mathrm{NF})-\kappa \mathrm{B}$, which is a master regulator of inflammation, cell proliferation, apoptosis and resistance in cells (5). NF- $\mathrm{B}$ regulates the expression of genes involved in the suppression of the apoptotic response, and is responsible for tumor cell survival (6). Additionally, Cur is also known to downregulate the intracellular levels of three major ABC drug transporters, P-gp, MDR-associated protein 1 (MRP1) and ABCG2, which are important in MDR (7-9). In spite of these various promising therapeutic applications of Cur, its therapeutic efficacy is limited due to its markedly poor water solubility, and consequently, remarkably low systemic concentrations are achieved when Cur is consumed orally (4). In addition, Cur suffers from chemical instability in the gastrointestinal tract (4). Cur is also known to be photosensitive, thus requiring careful handling (4). Cur is a substrate of P-gp, which implies that Cur can be pumped out of tumor cells by $\mathrm{P}-\mathrm{gp}$, leading to a reduction in drug accumulation in tumor cells (10). In spite of numerous formulations challenges, several formulation strategies, including nanoparticles, liposomes, complexation with phospholipids and cyclodextrins, and solid dispersions, are being developed to improve Cur's bioavailability (11-13).

Several absorption enhancers have also been used to improve Cur's bioavailability (14). Piperine (Pip) has been reported to enhance the bioavailability of Cur both in preclinical studies and in studies on human volunteers (15). Pip inhibits P-gp-mediated efflux in Caco-2 cells and CYP3A4-mediated drug metabolism (16). Pip reduces the ATPase activity of P-gp at high concentrations, while stimulates it at low 
concentrations (17). Pip can reverse MDR in short- and long-term treatments, and may improve the outcome of chemotherapy by inhibiting P-gp, MRP1 and breast cancer resistance protein effectively by downregulating the expression of these transporter genes (18). A marketed product available in combination with Pip is BioPerine ${ }^{\circledR}$ capsules (Sabinsa Corporation, East Windsor, NJ, USA). Despite its pharmacological activity and safety, this molecule still remains overlooked due to the lack of a suitable delivery system that can result in adequate therapeutic levels in vivo (14).

Several non-ionic surfactants are able to reverse P-gp-mediated MDR. For example, Pluronic sensitizes MDR tumors by inhibiting the P-gp drug efflux system through ATP depletion $(19,20)$. Tocopheryl polyethylene glycol succinate (TPGS), a water-soluble succinate ester of vitamin $\mathrm{E}$, can inhibit P-gp-mediated drug efflux and increase the oral bioavailability of anticancer drugs (21). Brij molecules have also shown efflux pump inhibitory activity. Dong et al prepared doxorubicin- and paclitaxel-loaded nanoparticles using Brij 78 as an emulsifying agent to overcome MDR by inhibiting P-gp and depleting ATP (22). Our laboratory has previously identified the structures of Brij required for overcoming MDR, and observed that Brij 78 and Brij 97 could decrease intracellular ATP levels and inhibit the ATPase activity of P-gp in MDR cells (23).

It was hypothesized that combining multiple strategies for overcoming drug resistance could improve the efficacy of antitumor drugs. The present study investigated a new co-delivery system of solid lipid nanoparticles (SLNs) with TPGS and Brij 78 to allow the anticancer drug Cur and the P-gp modulator Pip to overcome tumor drug resistance. (Cur+Pip)-SLNs were prepared by the emulsification evaporation-low temperature solidification method. In addition to formulation design and optimization, the physicochemical characterization, encapsulation efficiency (EE) and in vitro release behavior of (Cur+Pip)-SLNs were further investigated. In order to understand the efficacy of overcoming MDR in tumor cells, cytotoxicity assay and cell uptake experiments of (Cur+Pip)-SLNs were conducted on the paclitaxel-resistant human ovarian carcinoma cell line A2780/Taxol.

\section{Materials and methods}

Chemicals and reagents. Cur (purity 95\%) and Pip (purity 98\%) were obtained from Shaanxi Huike Botanical Development Co., Ltd. (Xi'an, China). Standard substances of Cur and Pip were supplied by the National Institutes for Food and Drug Control (Beijing, China). Glycerol monostearate was a gift of Gattefossé (Lyon, France). TPGS was purchased from Xi'an Healthful Biotechnology Co., Ltd. (Xi'an, China). Brij 78 was purchased from Sigma-Aldrich (Merck Millipore, Darmstadt, Germany). Oleic acid was purchased from Sinopharm Chemical Reagent Co., Ltd. (Shanghai, China). Soya lecithin was purchased from Aobox Biotechnology Co., Ltd. (Beijing, China). Sephadex G-50 was obtained from Pharmacia Biotech (GE Healthcare Life Sciences, Uppsala, Sweden). Verapamil hydrochloride was purchased from Shanghai Harvest Pharmaceutical Co., Ltd. (Shanghai, China).

RPMI-1640 medium was obtained from Thermo Fisher Scientific, Inc. (Waltham, MA, USA). Penicillin-streptomycin, fetal bovine serum (FBS) and PBS were purchased from Beijing Solarbio Science \& Technology Co., Ltd. (Beijing, China). Trypsin-EDTA was purchased from Gibco (Thermo Fisher Scientific, Inc.). MTT and rhodamine (Rh) 123 were obtained from Sigma-Aldrich (Merck Millipore). All solvents used in the present study were of high-performance liquid chromatography (HPLC) grade, and were used without further purification. Water was purified by distillation and deionization.

Preparation of (Cur+Pip)-SLNs, Cur-SLNs and blank SLNs. (Cur+Pip)-SLNs were prepared by the emulsification evaporation-low temperature solidification method (24). In brief, oleic acid, glycerol monostearate and lecithin were dissolved in ethyl acetate by ultrasonication at $50^{\circ} \mathrm{C}$ as the oil phase, and then the drugs in ethanol were added into the oil phase to obtain the organic phase containing Cur and Pip. The aqueous phase was prepared by adding TPGS and Brij 78 to deionized water under stirring for $10 \mathrm{~min}$ at $40^{\circ} \mathrm{C}$. Subsequently, the organic and aqueous phases were heated individually to $50-55^{\circ} \mathrm{C}$ for 2-4 min, and then the organic phase was injected into the aqueous phase. The suspension was magnetically stirred at $50^{\circ} \mathrm{C}$ for $20 \mathrm{~min}$ to remove the organic solvent. Finally, the suspension was cooled in an ice bath to form (Cur+Pip)-SLNs. The Cur-SLNs and blank SLNs were prepared following the same procedure, with the exception of adding only Cur or neither drug, respectively. These formulations were stored in a refrigerator at $4^{\circ} \mathrm{C}$ for further analysis. The drug content was determined by HPLC following the disruption of SLNs in acetonitrile.

Characterization of (Cur+Pip)-SLNs. To measure the EE of Cur and Pip, (Cur+Pip)-SLNs and Cur-SLNs were analyzed in a Sephadex G-50 column. In total, $0.2 \mathrm{ml}$ sample of (Cur+Pip)-SLNs was added to the column, and then eluted with deionized water. The unencapsulated Cur and Pip that remained in the gel were equilibrated with $30 \%$ ethanol. (Cur+Pip)-SLNs collected from the first $10 \mathrm{ml}$ eluent were destroyed with the mobile phase by ultrasonication for $\sim 10 \mathrm{~min}$. Another $0.2 \mathrm{ml}$ sample of (Cur+Pip)-SLNs was diluted with the same volume of mobile phase. The quantity of Cur and Pip loaded was determined by HPLC. EE was calculated according to the following formula: $\mathrm{EE}=\left(\mathrm{W}_{\mathrm{i}} / \mathrm{W}_{\text {total }}\right) \times 100 \%$, where $\mathrm{W}_{\mathrm{i}}$ is the determined quantity of Cur and Pip in the SLNs suspensions subsequent to passing over the Sephadex G-50 column, and $\mathrm{W}_{\text {total }}$ is the determined quantity of Cur and Pip in the SLNs suspensions prior to passing over the Sephadex G-50 column.

The contents of Cur and Pip were determined by HPLC analysis in a system equipped with Millennium 32 software (Waters Corporation, Milford, MA, USA), a 486 Tunable UV/ Visible Absorbance Detector (Waters Corporation) and a 510 HPLC Pump (Waters Corporation). The drug was separated using a Diamonsil ${ }^{\circledR} \mathrm{C} 18$ column $(200 \times 4.6 \mathrm{~mm}, 5 \mu \mathrm{m}$; Dikma Technologies Inc., Beijing, China). A 50:35:15 v/v/v methanol:acetonitrile:deionized water mixture was used as a the mobile phase, at a flow rate of $1.0 \mathrm{ml} / \mathrm{min}$ and a temperature of $25^{\circ} \mathrm{C}$. The run time was $6 \mathrm{~min}$ for each sample. Detection was monitored at a wavelength of 425 and $343 \mathrm{~nm}$ for Cur and Pip, respectively. 
The drug concentrations of Cur and Pip were calculated from calibration curves. The assay was linear over the tested concentration range of $0.5-20 \mu \mathrm{g} / \mathrm{ml}(\mathrm{r}=0.9998)$ for Cur and $0.2-10 \mu \mathrm{g} / \mathrm{ml}(\mathrm{r}=0.9998)$ for Pip. There was no interference from the excipients of the drugs in the assay. The lower limit of quantification was 0.025 and $0.030 \mu \mathrm{g} / \mathrm{ml}$ for Cur and Pip, respectively, which could be measured with acceptable accuracy and precision. The inter- and intra-day variance of the method was within the acceptable range of $<2 \%$.

Particle size, polydispersity index and zeta potential of (Cur+Pip)-SLNs were measured by dynamic light scattering using a Nano ZS90 Zetasizer (Malvern Instruments Inc, Westborough, MA, USA). The samples were prepared by diluting the (Cur+Pip)-SLN suspension with deionized water, and the data were obtained from the average of three measurements. The average was calculated by dividing the total value by the number of samples. The size and morphology of (Cur+Pip)-SLNs were observed using transmission electron microscopy (TEM) (H-7650; Hitachi, Ltd., Tokyo, Japan). For TEM analysis, a drop of (Cur+Pip)-SLNs suspension was placed into a copper grid, air-dried and stained by adding a drop of $1 \%$ uranyl acetate solution for contrast enhancement. The grid was kept at room temperature to ensure dryness and then observed under TEM.

The release rate of Cur and Pip from the SLNs in vitro was measured by dialyzing against release medium (PBS $\mathrm{pH} 7.4$ or 5.5 containing $25 \% \mathrm{v} / \mathrm{v}$ ethanol). In total, $1 \mathrm{ml}$ drug-loaded SLNs was placed into a dialysis tube (molecular weight cut-off, 14,000 kDa; Viskase Co., Osceola, AR, USA), and the end sealed of the dialysis tube was immersed fully in $200 \mathrm{ml}$ release medium in a beaker. The beakers were placed in an incubator at $37 \pm 0.5^{\circ} \mathrm{C}$ and agitated horizontally at a speed of $90 \mathrm{rpm}$. At the designated time intervals, $1 \mathrm{ml}$ release medium was withdrawn and replaced with an equal volume of fresh release medium to maintain the sink conditions. In in vitro release experiments, the solubility of a drug is $>5$-fold larger than the release of drug concentration, which is regarded as reaching the sink conditions. The solution was transferred into an HPLC vial upon filtering through a $0.45-\mu \mathrm{m}$ syringe filter. The analysis procedure was similar to that conducted for the determination of the EE percentage. The release rate was calculated with the formula: Release rate $=\left(\mathrm{W}_{\mathrm{i}} / \mathrm{W}_{\text {total }}\right) \times 100 \%$, where $\mathrm{W}_{\mathrm{i}}$ is the determined quantity of Cur and Pip in the release medium at the designated time point, and $\mathrm{W}_{\text {total }}$ is the total quantity of Cur and Pip in an equivalent volume of SLN suspension prior to the release experiment. All the samples were carefully protected from the light during the experiment. All the release experiments were repeated in triplicate.

Cell cultures. The paclitaxel-resistant human ovarian carcinoma cell line A2780/Taxol was kindly provided by Department of Gynecology and Obstetrics, The First Affiliated Hospital of Harbin Medical University (Harbin, China). A2780/Taxol cells were grown using $75-\mathrm{cm}^{3}$ flasks in a humidified $5 \% \mathrm{CO}_{2} / 95 \%$ atmosphere in an incubator at $37^{\circ} \mathrm{C}$ in RPMI-1640 medium supplemented with 10\% FBS, $100 \mathrm{U} / \mathrm{ml}$ penicillin and $100 \mathrm{mg} / \mathrm{ml}$ streptomycin. Cells grown to confluence were subcultured every other day upon trypsinization with $0.25 \%$ trypsin-EDTA.
Cytotoxicity assay in A2780/Taxol cells. The in vitro antitumor activity of drug-loaded SLNs, free drugs and excipients was determined by MTT assay as follows: Briefly, A2780/Taxol cells in logarithmic growth phase were seeded in 96-well plates at a density of 5,000 cells/well. Following attachment overnight, the culture medium in each well was carefully replaced with $100 \mu \mathrm{l}$ fresh medium containing treatments. The treatments included (Cur+Pip)-SLNs, Cur-SLNs, free Cur solution, free Pip solution, combination of Cur and Pip solution, TPGS, Brij 78 and blank SLNs.

After incubation for $24 \mathrm{~h}$, the medium was removed and the cells were washed with PBS. Then, the viability of the cells was determined by MTT assay. For that purpose, $50 \mu \mathrm{l}$ $5 \mathrm{mg} / \mathrm{ml} \mathrm{MTT} \mathrm{dissolved} \mathrm{in} \mathrm{PBS} \mathrm{was} \mathrm{added} \mathrm{to} \mathrm{each} \mathrm{well.} \mathrm{The}$ plates were incubated for an additional $4 \mathrm{~h}$ at $37^{\circ} \mathrm{C}$, and then the medium was discarded. Thereafter, $100 \mu \mathrm{l}$ dimethyl sulfoxide was added to each well to dissolve the formazan crystals. The absorbance of each well was assessed on a microplate reader at a wavelength of $490 \mathrm{~nm}$. The spectrophotometer was calibrated to an absorbance value of 0 using culture medium without cells, and cells containing culture medium without nanoparticles or drugs were treated as controls. The relative cell viability was calculated by $(\mathrm{A})_{\text {test }} /(\mathrm{A})_{\text {control }}$, where $(\mathrm{A})_{\text {test }}$ and (A) control were the average absorbance of the test and control samples, respectively.

Rh efflux assay. The accumulation of Rh 123 in A2780/Taxol cells was first detected by confocal laser scanning microscopy (CLSM). A2780/Taxol cells were seeded onto round glass coverslips in 6-well plates for $24 \mathrm{~h}$. Once the medium had been removed, the cells were treated with (Cur+Pip)-SLNs, Cur-SLNs, free Cur solution, free Pip solution, Cur+Pip solution, TPGS, Brij 78 and blank SLNs separately for $4 \mathrm{~h}$ at $37^{\circ} \mathrm{C}$ and $5 \% \mathrm{CO}_{2}$. Then, $\mathrm{Rh} 123(5 \mu \mathrm{g} / \mathrm{ml})$ was added, and the cells were incubated for an additional $1 \mathrm{~h}$. Following this incubation period, the cells were collected and washed twice with ice-cold PBS buffer ( $\mathrm{pH}$ 7.4). Coverslips were placed onto glass microscope slides and inspected by CLSM (BX40; Olympus Corporation, Tokyo, Japan).

Statistical analysis. The mean and standard deviation (SD) were determined for each treatment group. Statistical analysis was performed using a Student's $t$-test and Microsoft Office 2013 software (Microsoft Corporation, Redmond, WA, USA). $\mathrm{P}<0.05$ was considered to indicate a statistically significant difference.

\section{Results and Discussion}

EE of (Cur+Pip)-SLNs. Compared with polymeric nanoparticles, SLNs attracted more attention recently, since SLNs could not only avoid the disadvantages of other colloidal carriers, but also have excellent inherent properties, including controlled drug release and drug targeting, high drug load capacity, increased physical stability and low toxicity (24). Therefore, the development of (Cur+Pip)-SLNs should be a worthwhile and promising strategy. In the present study, (Cur+Pip)-SLNs were prepared by the emulsification evaporation-low temperature solidification method. The average EE values of Cur and Pip were $87.4 \pm 0.6$ and $14.7 \pm 0.2 \%$, 
A

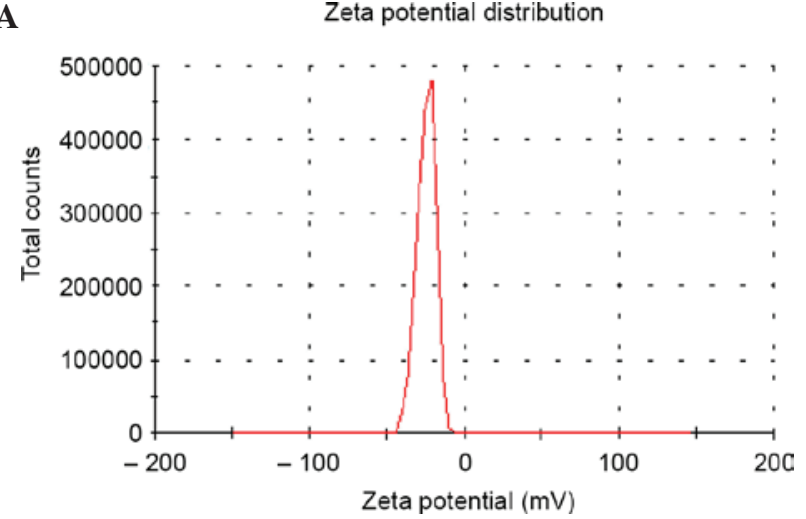

B

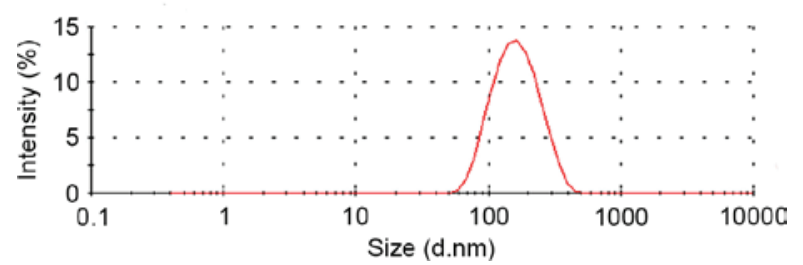

Figure 1. (A) Zeta potential and (B) size distribution of curcumin and piperine encapsulated in solid lipid nanoparticles. $d$, diameter.

respectively, with a drug load of $19.56 \pm 0.18 \mu \mathrm{g} / \mathrm{mg}$ for Cur and $3.26 \pm 0.05 \mu \mathrm{g} / \mathrm{mg}$ for Pip. The EE and drug load of three batches of (Cur+Pip)-SLNs had no significant difference, with a relative SD of $0.6 \%$ for Cur and $0.2 \%$ for Pip, which demonstrated that the preparation process was reproducible and stable.

Zeta potential and particle size. The zeta potential, particle size and size distribution of (Cur+Pip)-SLNs were characterized with a Nano ZS90 Zetasizer. Zeta potential is an important parameter used to predict the physical stability of nanoparticles. A high zeta potential value indicates increased stability of the system, since it could provide a repelling force between the nanoparticles (25). As shown in Fig. 1A, (Cur+Pip)-SLNs had a relatively high negative zeta potential of $\sim 20 \mathrm{mV}$. (Cur+Pip)-SLNs exhibited a mean particle diameter of $\sim 130.8 \mathrm{~nm}$, with a unimodal size distribution and a polydispersity index of 0.152 (Fig. 1B). The polydispersity index is an parameter used to represent the distribution of nanoparticles, and indicates a low aggregation of particles when its value is $<0.5$ (26).

Compared to free Pip solution (Fig. 2A, middle) or free Cur solution (Fig. 2A, right), (Cur+Pip)-SLNs (Fig. 2A, left) could be dispersed homogeneously into water without apparent drug sediments. In addition, a smooth sphere morphology and uniform size distribution of (Cur+Pip)-SLNs were observed by TEM (Fig. 2B).

In vitro drug release studies. Fig. 3 represents the in vitro cumulative release profiles of Cur and Pip from (Cur+Pip)-SLNs in PBS. PBS (pH 5.5) was used in the present study to simulate the internal environment of tumor cells, since the $\mathrm{pH}$ in tumors is lower than that in normal tissue (27). PBS (pH 7.4) was selected to simulate the environment of blood (27). Due to the poor water solubility of Cur and Pip, it is difficult to maintain
A

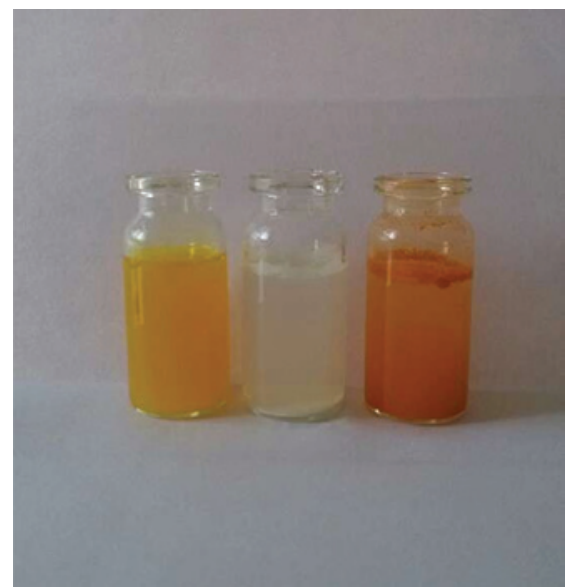

B

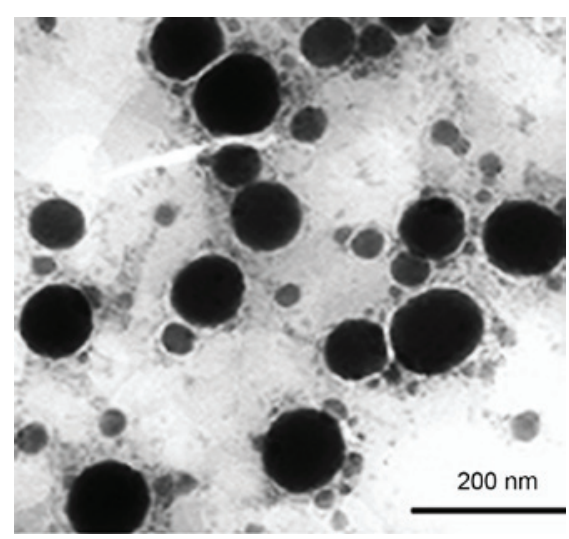

Figure 2. (A) Photograph showing (Cur+Pip)-SLNs (left), free Pip in water (middle) and free Cur in water (right). (B) Transmission electron microscopy image of (Cur+Pip)-SLNs. Cur, curcumin; Pip, piperine; SLN, solid lipid nanoparticle.

a good sink condition when carrying out the in vitro release experiments. In the present study, $25 \% \mathrm{v} / \mathrm{v}$ ethanol was added to the release medium to maintain sink conditions, in which the solubility of Cur and Pip was 0.265 and $0.195 \mathrm{mg} / \mathrm{ml}$, respectively. Approximately $71.5 \%$ of Cur was released at $96 \mathrm{~h}$, and $\sim 89 \%$ of Pip was released in $24 \mathrm{~h}$ in PBS pH 7.4. In addition, these profiles exhibited a burst release of $\sim 36 \%$ during the first $2 \mathrm{~h}$ of release, which probably is due to the non-encapsulation of the drugs. In addition, the drug cumulative release percentage displayed a slight $\mathrm{pH}$ dependence. For instance, the Cur cumulative release percentage at $96 \mathrm{~h}$ was $71.5 \%$ at $\mathrm{pH} 7.4$, while it was $83.7 \%$ at $\mathrm{pH} 5.5$, which indicated that the SLNs released drug more rapidly in the acidic environment of $\mathrm{pH} 5.5$ than in a $\mathrm{pH} 7.4$ environment.

Cytotoxicity assay in A2780/Taxol cells. Firstly, the cytotoxicity/cell viability effect of Pip was investigated in A2780/Taxol cells. When treated with Pip at concentrations of $1-50 \mu \mathrm{M}$, the cytotoxicity on A2780/Taxol cells was negligible (Fig. 4). Since the half maximal inhibitory concentration of Pip (318 $\mu \mathrm{M})$ in A2780/Taxol cells did not change significantly following the addition of verapamil [an inhibitor of P-gp (28)], it was confirmed that Pip was not the substrate of P-gp. Singh et al had studied the cytotoxicity of Pip in the MCF-7 (a breast cancer cell line) and MDCK cell lines (28). Their results revealed that Pip did not exert any undesirable effect at concentrations $\leq 100 \mu \mathrm{M}$. 
A

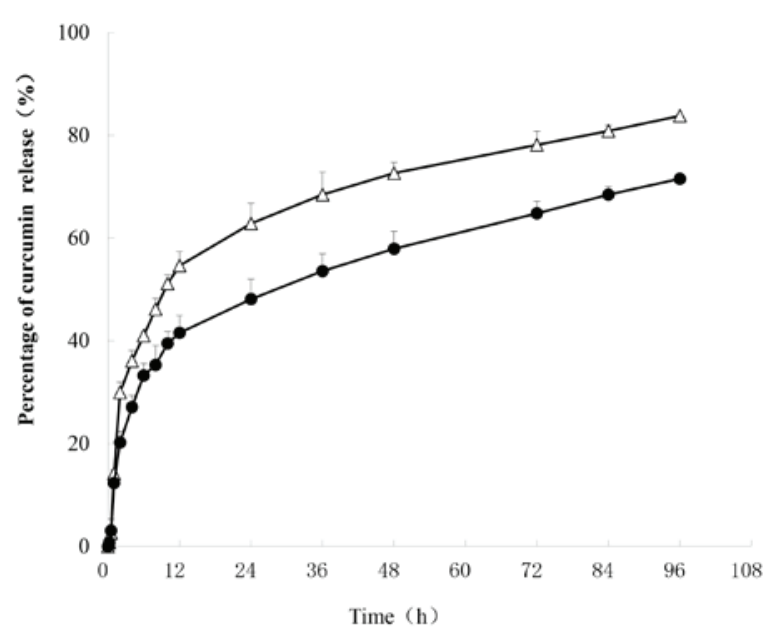

B

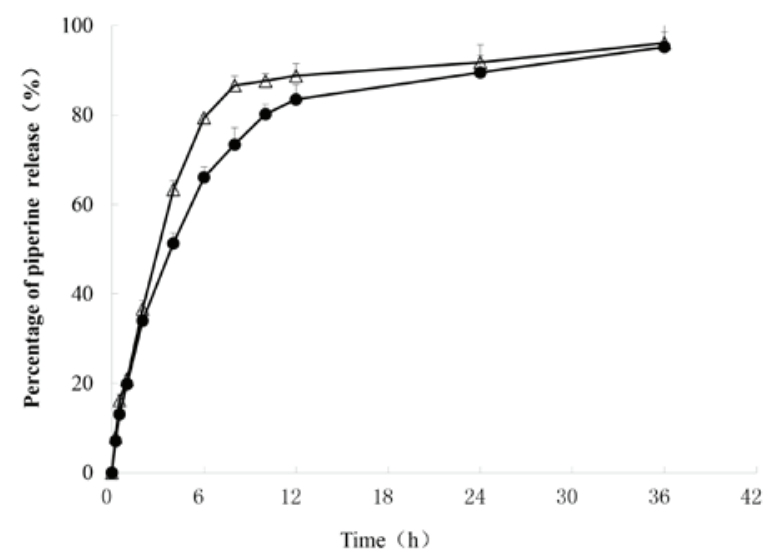

Figure 3. Cumulative release profiles of (A) Cur and (B) Pip from (Cur+Pip)-SLNs at pH $5.5(\Delta)$ and $7.4(\bullet)$. Data are represented as the mean + standard deviation, $n=3$. Cur, curcumin; Pip, piperine; SLN, solid lipid nanoparticle.

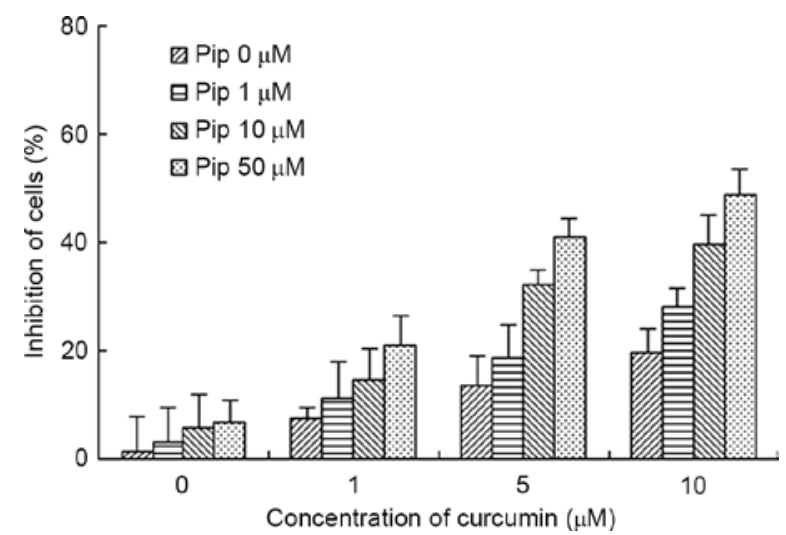

Figure 4. Effect of Pip and curcumin combination on the inhibition of cell growth in A2780/Taxol cells. Data are represented as the mean + standard deviation, $\mathrm{n}=6$. Pip, piperine.

Secondly, the effect of Pip on Cur-treated cells' viability was investigated in A2780/Taxol cells. With increased concentration of Pip, the anti-proliferative effect of Cur was

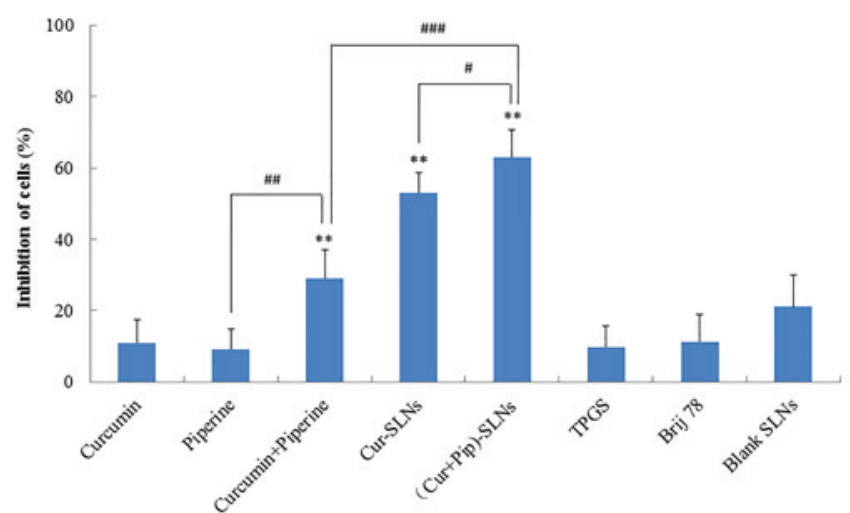

Figure 5. Cytotoxicity of different formulations in A2780/Taxol cells following treatment. Data are represented as the mean + standard deviation, $\mathrm{n}=6,{ }^{* *} \mathrm{P}<0.01$ compared with Cur; ${ }^{*} \mathrm{P}<0.05$ compared with Cur-SLNs; ${ }^{\# \#} \mathrm{P}<0.01$ compared with Pip; ${ }^{\# \# \#} \mathrm{P}<0.01$ compared with Cur + Pip. Cur, curcumin; Pip, piperine; SLN, solid lipid nanoparticle; TPGS, tocopheryl polyethylene glycol succinate.
A

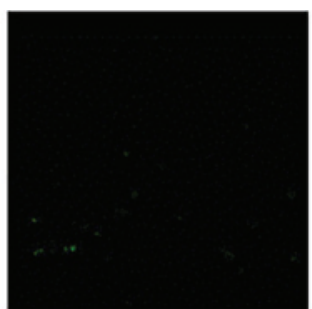

C

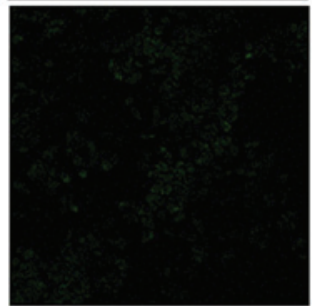

E

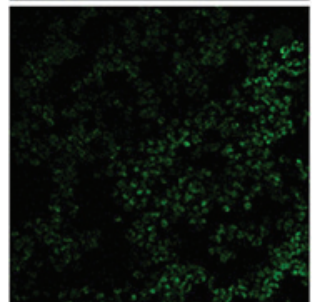

G

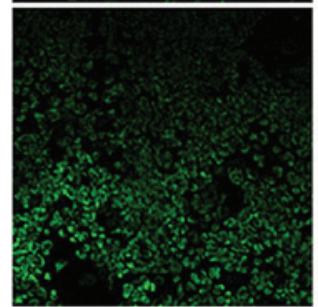

B

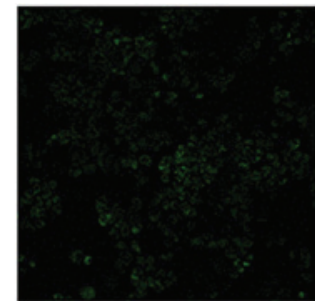

D

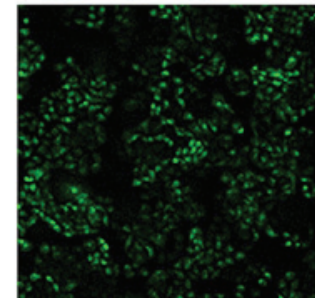

F

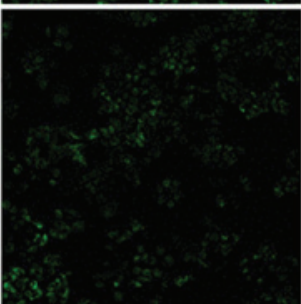

H

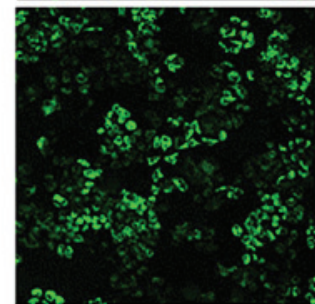

Figure 6. Confocal microscopic images of A2780/Taxol cells incubated with (A) medium (control), (B) free Pip solution, (C) free Cur solution, (D) tocopheryl polyethylene glycol succinate, (E) Brij 78, (F) blank SLNs, (G) Cur-SLNs and (H) (Cur+Pip)-SLNs. The green color represents rhodamine 123 fluorescence. Magnification, x20. Cur, curcumin; Pip, piperine; SLN, solid lipid nanoparticle.

significantly enhanced. Upon treatment of the cells with Cur and Pip (10 and $50 \mu \mathrm{M}$, respectively), the inhibition rate of the cells was $59.90 \pm 8.40 \%$, which was significantly higher than 
that observed with Cur $(25.85 \pm 5.74 \%)$ and Pip $(6.87 \pm 4.52 \%)$ treatment alone (Fig. 4). These results indicate that Pip, an inhibitor of P-gp, can enhance the anti-proliferative effect of Cur, which is a substrate of P-gp (10).

TPGS and Brij 78 can inhibit P-gp, thus sensitizing MDR cells (23). In the present study, the effect of SLNs with TPGS and Brij 78 on sensitizing MDR cells was investigated in A2780/Taxol cells. As shown in Fig. 5, cell inhibition following treatment with Cur-SLNs or (Cur+Pip)-SLNs was higher than that observed following treatment with free Cur or Cur-Pip solutions $(\mathrm{P}<0.01)$. By contrast, blank SLNs, free Brij or TPGS did not apparently inhibit cell growth. It was demonstrated that SLNs with TPGS and Brij 78 could sensitize MDR cells (21). SLNs serve as potential anticancer drug delivery nanocarriers, since they exhibit great superiority to modulate drug release, improve anticancer activity and overcome MDR. SLNs have also been shown to increase the cellular accumulation of drugs, since they could decrease the resistance of P-gp-expressing cells.

When treated with (Cur+Pip)-SLNs, MDR cells exhibited the highest response to its cytotoxic action $(\mathrm{P}<0.01)$, which presumably resulted from the synergistic effect of Cur, Pip and Pip-mediated P-gp inhibition in A2780/Taxol cells, thus amplifying the Cur-induced cytotoxicity and overcoming MDR in tumor cells. The effect of Pip on increasing the intracellular Cur concentration was the key to the enhancement of the cytotoxicity in resistant cells.

P-gp serves a significant role in the bioavailability of several drugs, mainly cytotoxic hydrophobic and anticancerous drugs (2). In the present study, although the drug load of Pip in (Cur+Pip)-SLNs $(3.26 \pm 0.05 \mu \mathrm{g} / \mathrm{mg})$ was not very high, the enhancement of cytotoxicity exerted by Cur was remarkable, since Pip is known to be a strong inhibitor of P-gp (28).

Rh efflux assay. MDR is a major clinical problem that reduces the efficacy of a large number of chemotherapeutic agents. The most classical mechanism of MDR is the overexpression of P-gp, which can successfully pump out multiple antineoplastic agents from cells, thereby decreasing their intracellular accumulation and leading to drug resistance (2). To investigate whether (Cur+Pip)-SLNs inhibit the function of P-gp, the intracellular accumulation of $\mathrm{Rh} 123$, which is a substrate of $\mathrm{P}$-gp, in the presence or absence of SLNs, was examined using A2780/Taxol cells. It was reported that P-gp function was significantly correlated with Rh 123 efflux, and that inhibition of P-gp could result in increased intracellular accumulation of Rh 123 (29). Thus, Rh 123 could be a fluorescent dye used to monitor P-gp function (30). As shown in Fig. 6A, Rh 123 fluorescence was scarcely detected in A2780/Taxol cells treated with free $\mathrm{Rh} 123$, which indicated a strong efflux phenomenon of Rh 123 in resistant cells. The inhibited Rh 123 efflux in A2780/Taxol cells treated with free Cur was hardly observed. However, treatment with Pip could cause apparently accumulation of the fluorescent dye Rh 123 in the cells (Fig. 6C). It has been reported that Pip could be used for the design and development of safe non-toxic P-gp inhibitors, since the structural features of Pip could bind in the vicinity of the ATP binding site (28). This suggests that Pip could be used for the development of the next generation of P-gp inhibitors. In addition, treatment with the surfactants TPGS and Brij 78 could also cause accumulation of Rh 123 (Fig. 6D and E). Recently, surfactants such as TPGS have been noticed to modulate efflux pump activity $(21,31)$. These surfactants are used as excipients in the preparation of nanoparticles, and are aimed at specifically targeting the therapeutic drug to tumors and overcoming MDR.

In the present study, A2780/Taxol cells treated with (Cur+Pip)-SLNs exhibited a higher uptake than those treated with free Cur, free Pip, excipients and control nanoparticles. The increased accumulation of Rh 123 within A2780/Taxol cells may be a result of decreased expression of P-gp or inhibited function of P-gp. In our previous studies $(31,32)$, western blot analyses were performed to assess the effect of Brij 78 and TPGS on the protein expression levels of P-gp. Upon pretreatment with Brij 78 or TPGS, the expression level of P-gp protein was not significantly altered compared with that in control H460/TaxR cells. These results indicate that inhibiting the expression of P-gp is not involved in the reversal of P-gp MDR by Brij 78 or TPGS.

The present study suggests that there are at least two major reasons for the enhanced uptake of Rh 123 in P-gp-mediated resistant cells: i) Increased Rh 123 uptake by endocytosis of SLNs, which helps to partially bypass P-gp; and ii) decreased efflux of Rh 123 through inhibition of P-gp function caused by Pip, TPGS or Brij 78.

In conclusion, SLNs with TPGS and Brij 78 co-delivering Cur and Pip were designed and studied to overcome MDR in A2780/Taxol cells in the present study. (Cur+Pip)-SLNs were successfully prepared and optimized by the emulsification evaporation-low temperature solidification method. (Cur+Pip)-SLNs exhibited high cytotoxicity and allowed efficient intracellular drug delivery. TPGS and Brij 78 also serve an important role in the inhibition of P-gp. The combination of Cur and Pip, when administered in SLNs formulations, resulted in a significant enhancement in cytotoxicity in drug-resistant A2780/Taxol cells. This dual inhibitory strategy can have a significant potential in the clinical management of MDR in cancer. Future in vivo studies in human tumor xenograft models will further validate this hypothesis.

\section{Acknowledgements}

The present study was financially supported by the National Natural Science Foundation of China (Beijing, China; grant no. 81302705) and the Returned Overseas Foundation of Heilongjiang Province of China (Harbin, China; grant no. LC201432).

The authors would like to acknowledge Dr Xiaohan Tang (Department of Gynecology and Obstetrics, The First Affiliated Hospital of Harbin Medical University, Harbin, China) for kindly providing the A2780/Taxol cells.

\section{References}

1. Fodale V, Pierobon M, Liotta L and Petricoin E: Mechanism of cell adaptation: When and how do cancer cells develop chemoresistance? Cancer J 17: 89-95, 2011.

2. Kathawala RJ, Gupta P, Ashby CR Jr and Chen ZS: The modulation of $\mathrm{ABC}$ transporter-mediated multidrug resistance in cancer: $\mathrm{A}$ review of the past decade. Drug Resist Updat 18: 1-17, 2015. 
3. Shapira A, Livney YD, Broxterman HJ and Assaraf YG: Nanomedicine for targeted cancer therapy: Towards the overcoming of drug resistance. Drug Resist Updat 14: 150-163, 2011.

4. Anand P, Kunnumakkara AB, Newman RA and Aggarwal BB Bioavailability of curcumin: Problems and promises. Mo Pharm 4: 807-818, 2007.

5. Aggarwal BB, Shishodia S, Takada Y, Banerjee S, Newman RA, Bueso-Ramos CE and Price JE: Curcumin suppresses the paclitaxel-induced nuclear factor-kappaB pathway in breast cancer cells and inhibits lung metastasis of human breast cancer in nude mice. Clin Cancer Res 11: 7490-7498, 2005.

6. Aggarwal BB: Nuclear factor-kappaB: The enemy within. Cancer Cell 6: 203-208, 2004.

7. Limtrakul P, Chearwae W, Shukla S, Phisalphong C and Ambudkar SV: Modulation of function of three ABC drug transporters, P-glycoprotein (ABCB1), mitoxantrone resistance protein $(\mathrm{ABCG})$ and multidrug resistance protein 1 (ABCC1) by tetrahydrocurcumin, a major metabolite of curcumin. Mol Cell Biochem 296: 85-95, 2007.

8. Chearwae W, Wu CP, Chu HY, Lee TR, Ambudkar SV and Limtrakul P: Curcuminoids purified from turmeric powder modulate the function of human multidrug resistance protein 1 (ABCC1). Cancer Chemother Pharmacol 57: 376-388, 2006.

9. Ganta S and Amiji M: Coadministration of Paclitaxel and curcumin in nanoemulsion formulations to overcome multidrug resistance in tumor cells. Mol Pharm 6: 928-939, 2009.

10. Romiti N, Tongiani R, Cervelli F and Chieli E: Effects of curcumin on P-glycoprotein in primary cultures of rat hepatocytes. Life Sci 62: 2349-2358, 1998.

11. Bisht S, Feldmann G, Soni S, Ravi R, Karikar C, Maitra A and Maitra A: Polymeric nanoparticle-encapsulated curcumin ('nanocurcumin'): A novel strategy for human cancer therapy. J Nanobiotechnology 5: 3, 2007.

12. Maiti K, Mukherjee K, Gantait A, Saha BP and Mukherjee PK: Curcumin-phospholipid complex: Preparation, therapeutic evaluation and pharmacokinetic study in rats. Int $\mathbf{J}$ Pharm 330 : $155-163,2007$.

13. Tiyaboonchai $\mathrm{W}$, Tungpradit $\mathrm{W}$ and Plianbangchang $\mathrm{P}$. Formulation and characterization of curcuminoids loaded solid lipid nanoparticles. Int J Pharm 337: 299-306, 2007.

14. Shaikh J, Ankola DD, Beniwal V, Singh D and Kumar MN Nanoparticle encapsulation improves oral bioavailability of curcumin by at least 9-fold when compared to curcumin administered with piperine as absorption enhancer. Eur J Pharm Sci 37: 223-230, 2009

15. Shoba G, Joy D, Joseph T, Majeed M, Rajendran R and Srinivas PS: Influence of piperine on the pharmacokinetics of curcumin in animals and human volunteers. Planta Med 64 353-356, 1998

16. Bhardwaj RK, Glaeser H, Becquemont L, Klotz U, Gupta SK and Fromm MF: Piperine, a major constituent of black pepper, inhibits human P-glycoprotein and CYP3A4. J Pharmacol Exp Ther 302: 645-650, 2002

17. Najar IA, Sachin BS, Sharma SC, Satti NK, Suri KA and Johri RK: Modulation of P-glycoprotein ATPase activity by some phytoconstituents. Phytother Res 24: 454-458, 2010.

18. Li S, Lei Y, Jia Y, Li N, Wink M and Ma Y: Piperine, a piperidine alkaloid from Piper nigrum re-sensitizes P-gp, MRP1 and BCRP dependent multidrug resistant cancer cells. Phytomedicine 19: 83-87, 2011.
19. Kabanov AV, Batrakova EV and Alakhov VY: An essential relationship between ATP depletion and chemosensitizing activity of Pluronic block copolymers. J Control Release 91: 75-83, 2003.

20. Batrakova EV, Li S, Brynskikh AM, Sharma AK, Li Y, Boska M, Gong N, Mosley RL, Alakhov VY, Gendelman HE and Kabanov AV: Effects of pluronic and doxorubicin on drug uptake, cellular metabolism, apoptosis and tumor inhibition in animal models of MDR cancers. J Control Release 143: 290-301, 2010.

21. Guo Y, Luo J, Tan S, Otieno BO and Zhang Z: The applications of Vitamin E TPGS in drug delivery. Eur J Pharm Sci 49: 175-186, 2013.

22. Dong X, Mattingly CA, Tseng MT, Cho MJ, Liu Y, Adams VR and Mumper RJ: Doxorubicin and paclitaxel-loaded lipid-based nanoparticles overcome multidrug resistance by inhibiting P-glycoprotein and depleting ATP. Cancer Res 69: 3918-3926, 2009.

23. Tang J, Wang Y, Wang D, Wang Y, Xu Z, Racette K and Liu F: Key structure of brij for overcoming multidrug resistance in cancer. Biomacromolecules 14: 424-430, 2013.

24. Patel BD, Modi RV, Thakkar NA, Patel AA and Thakkar PH: Development and characterization of solid lipid nanoparticles for enhancement of oral bioavailability of Raloxifene. J Pharm Bioallied Sci 4 (Suppl 1): S14-S16, 2012.

25. Loveless VS, Surdock CP and Bhattacharjee H: Evaluation of zeta-potential and particle size of technetium $99 \mathrm{mTc}$-sulfur colloid subsequent to the addition of lidocaine and sodium bicarbonate. J Nucl Med Technol 38: 49-52, 2010.

26. Yusuf M, Khan RA, Khan M and Ahmed B: Plausible antioxidant biomechanics and anticonvulsant pharmacological activity of brain-targeted $\beta$-carotene nanoparticles. Int J Nanomedicine 7: 4311-4321, 2012.

27. Raghunand N, He X, Van Sluis R, Mahoney B, Baggett B, Taylor CW, Paine-Murrieta G, Roe D, Bhujwalla ZM and Gillies RJ: Enhancement of chemotherapy by manipulation of tumour pH. Br J Cancer 80: 1005-1011, 1999.

28. Singh DV, Godbole MM and Misra K: A plausible explanation for enhanced bioavailability of P-gp substrates in presence of piperine: Simulation for next generation of P-gp inhibitors. J Mol Model 19: 227-238, 2013.

29. Szakács G, Paterson JK, Ludwig JA, Booth-Genthe C and Gottesman MM: Targeting multidrug resistance in cancer. Nat Rev Drug Discov 5: 219-234, 2006.

30. Eytan GD, Regev R, Oren G, Hurwitz CD and Assaraf YG: Efficiency of P-glycoprotein-mediated exclusion of rhodamine dyes from multidrug-resistant cells is determined by their passive transmembrane movement rate. Eur J Biochem 248: 104-112, 1997.

31. Tang J, Wang Y, Wang D, Xu Z, Racette K and Liu F: Key structure of brij for overcoming multidrug resistance in cancer. Biomacromolecules 14: 424-430, 2013.

32. Tang J, Fu Q, Wang Y, Racette K, Wang D and Liu F: Vitamin $\mathrm{E}$ reverses multidrug resistance in vitro and in vivo. Cancer Lett 336: 149-157, 2013. 